\title{
Design of a mega-sub-controlled building system under stochastic wind loads
}

\author{
M. Q. Feng $\dagger$ \& W. Chai \\ Department of Civil and Environmental Engineering, University of California, Irvine, CA 92717, USA
}

\begin{abstract}
Vibration control of high-rise buildings under wind loads with application of the mega-sub-control method is studied in this paper. A building with a megasub-configuration consists of two major structural components - a megastructure as the main structural frame and several sub-structures for residential and/or commercial usage. The authors have previously proposed a 'megasub-control method' in which the sub-structures are designed to serve as vibration control dampers. The control objective is to suppress certain critical building responses such as inter-story drifts of the mega-structure for the purpose of structural safety and acceleration response of the sub-structures for the purpose of protecting contents and improving human comfort. The feasibility of this method has been explored by the authors in previous publications. In this study, the procedure of optimally designing dynamic parameters of a megasub-controlled building under stochastic wind loads is developed, together with two possible structural configurations which provide a mega-sub-control mechanism. The mega-structure of a mega-sub-building is modeled as a cantilever beam to retain the dominant bending mode characteristics of highrise buildings, and the sub-structure as a shear building to retain the shear mode. The fluctuating wind speed is modeled as a non-white random process in both time and space domains. The power spectral density (PSD) of critical building responses is obtained using the random vibration theory. The mean square value (MSV) of those responses, as functions of the dynamic parameters including the stiffness and damping ratio of the sub-structures, are evaluated from their PSD by numerical integration in the frequency domain. The optimal values of the dynamic parameters are determined by minimizing the MSV of certain critical building responses. An example building is used to demonstrate the design procedure and the numerical simulation of the response quantities in the time domain is carried out to verify the MSV of the building responses obtained from the random vibration theory in the frequency domain. The results show that the proposed design procedure is suitable to apply to a mega-sub-building with different sub-structural configurations. The MSV obtained from the random vibration theory in the frequency domain and from the numerical simulation in the time domain exhibit an excellent agreement. It is also found that the megasub-control method is robust in the sense that slight change in the dynamic parameters affects the building's performance very little. With the design procedure developed, and the corresponding favorable building response demonstrated, this paper has enhanced the feasibility of application of the mega-sub-control method to actual high-rise buildings for wind vibration suppression. (C) 1997 Elsevier Science Ltd.
\end{abstract}

\section{INTRODUCTION}

Vibration control of high-rise buildings presents a unique civil engineering challenge. With a slender configuration, a high-rise building possesses a low fundamental frequency and hence is susceptible to

†Author to whom correspondence should be addressed. turbulent wind. Consequently, the building's capacity to resist not only seismic but also wind loads is critical for its structural integrity. Because of the prolonged duration and frequent occurrence, wind loads often cause serious serviceability problems. Excessive vibration resulting from strong gusty wind often makes occupants feel physically and psychologically uncomfortable and may damage the vibration sensitive 
contents as well. Therefore, wind excitation is usually a primary concern in the design of high-rise buildings.

Compared to a conventional frame building [Fig. 1(a)], the mega-sub-configuration [Fig. 1(b)] is gaining more popularity in design and construction of high-rise buildings. Typical examples include the Bank of China Building in Hong Kong and the Tokyo Metropolitan Government Building in Japan. A mega-sub-building consists of two major components - a mega-structure which is the main structural frame of the building and several sub-structures, each of which may contain several floors used for residential and/or commercial purposes. Unlike the mega-structure, which is required not only to support the self weight of the building but also to resist the external loads such as wind and earthquakes, a sub-structure is only required to support its own weight. This special configuration provides structural designers with an opportunity to design a substructure as a vibration control damper whose function is similar to a tuned-mass-damper (TMD) but without the requirement of additional structurally useless mass. The large mass ratio (usually $100 \%$ ) between the suband mega-structures makes the mega-sub-control much more effective than the TMD control. Feng and Mita ${ }^{1}$ were the first to propose the idea of utilizing substructures as dampers to control building vibration. A more detailed feasibility study has been conducted by Chai and Feng. ${ }^{2}$

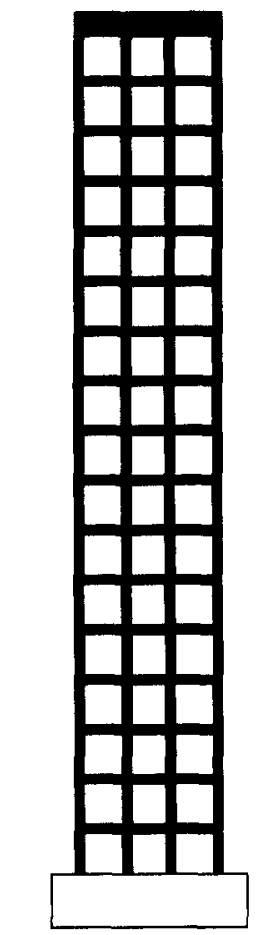

Conventional Building

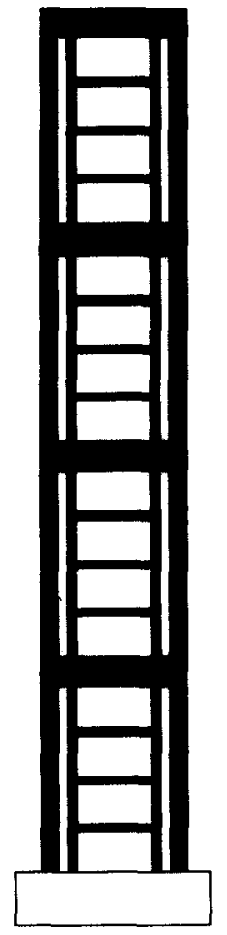

Mega-Sub Building

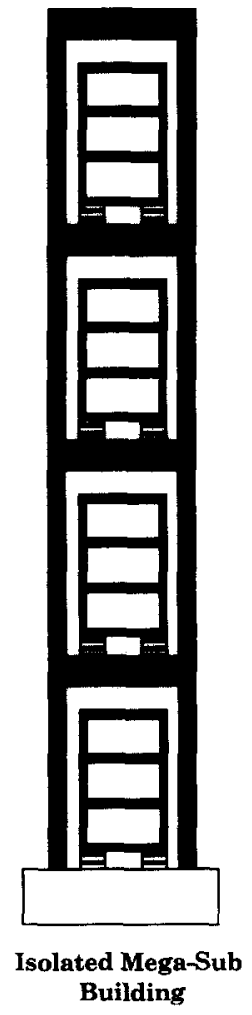

(c)
Fig. 1. Configurations of conventional building and megasub-buildings.
Unlike the TMD control in which the vibration of the mass damper itself does not need to be controlled as long as the stroke restriction is not exceeded, the objective of the mega-sub-control is to reduce the vibration of not only the mega-structure but also the sub-structures. In particular, the inter-story drifts of the mega-structure and the acceleration response of the sub-structures are considered as the critical responses needed to be controlled in order to enhance the structural safety and serviceability. Therefore, the design procedure for the TMD control cannot be directly applied to the design of the mega-sub-control. This study develops a design procedure for optimally determining the dynamic parameters such as the stiffness and damping ratios of sub-structures. Since the dynamic loading is the fluctuating component of wind force which is usually modeled as a stationary random process in both time and space domains, the application of random vibration theory is a proper approach. The dynamic characteristic of fluctuating wind is represented by its PSD which is defined as a function of the frequency and wave-number reflecting that the wind speed has both temporal and spatial fluctuations with the latter along the height of the building. The PSD function of the building response in the along-wind direction is obtained by applying the random vibration theory on the basis of the PSD function of the fluctuating component of wind speed and the frequency response functions of the mega-sub-building. It is then numerically integrated in the frequency domain to calculate the MSV of the response for the evaluation of the building's performance. The values of the dynamic parameters, including the stiffness and damping ratios of the sub-structures which result in the minimum MSV of the responses, are then searched.

Another possible type of mega-sub-configuration is also studied, in which each sub-structure is base-isolated from the mega-structure as shown in Fig. 1(c). The same procedure is applied to design the dynamic properties such as the stiffness and damping of the isolators as well.

\section{ANALYTICAL MODEL}

The configuration of a mega-sub-building is illustrated in Fig. 1(b), where the main frame is the mega-structure with several sub-structures attached, while each substructure usually contains several stories. For a high-rise building where bending is the dominant vibration mode, the mega-structure should be modeled as a cantilever beam. The beam is further discretized as a multi-degreeof-freedom (MDOF) system. A shear building model is correct for a sub-structure due to its less slender shape. Therefore, the analytical model of a mega-sub-controlled building can be obtained as shown in Fig. 2(a), and for the purpose of formulating the stiffness and damping matrices, it is further divided into a mega-frame and a sub-frame as shown in Figs 2(b) and (c), respectively. 


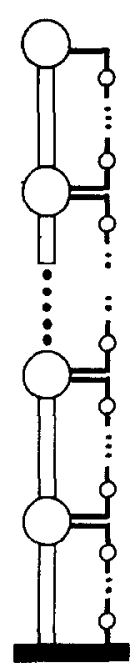

(a) Mega-Sub Frame

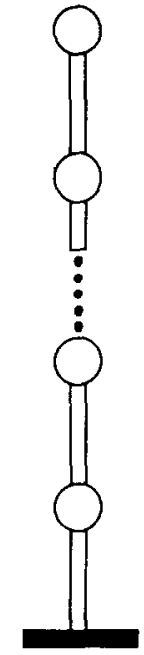

(b) Mega Frame

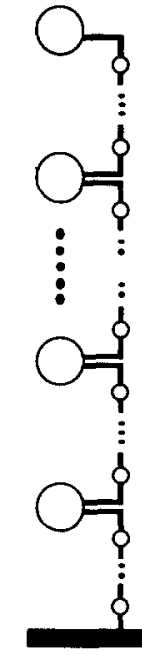

(c) Sub Frame
Fig. 2. Analytical model of mega-sub-building.

The mega-frame is modeled as a cantilever beam, and its stiffness matrix $\left[K^{\mathrm{m}}\right]$ is formed after the condensation operation $^{3}$ so that the rotational stiffness is expressed in terms of the translational stiffness. The mass matrix $\left[M^{\mathrm{m}}\right]$ is a diagonal matrix consisting of masses of the mega-structure. The damping matrix $\left[C^{\mathrm{m}}\right]$ is a linear combination of the mass and stiffness matrices generated by the following equation: ${ }^{3}$

$$
\left[C^{\mathrm{m}}\right]=\left[\boldsymbol{M}^{\mathrm{m}}\right] \sum_{i} a_{i}\left(\left[\boldsymbol{M}^{\mathrm{m}}\right]^{-1}\left[\boldsymbol{K}^{\mathrm{m}}\right]\right)^{i} \quad i=1,2, \ldots
$$

where $a_{i}=$ constant,

$$
\xi_{j}=\frac{1}{2 \omega_{j}} \sum_{i} a_{i} \omega_{j}^{2 i}
$$

is the damping ratio of the $j$ th mode of mega-frame and $\omega_{j}=$ the $j$ th natural frequency of mega-frame.

A sub-frame unit, consisting of two mega-floors and a sub-structure between them, is modeled as a shear building. Assembling all the unit stiffness matrices and moving all the rows and columns corresponding to the mega-floors to the top left portion of the assembled matrix, the stiffness matrix of the whole sub-frame $\left[K^{\mathrm{s}}\right]$ is formed. In the process of acquiring the damping matrix of a sub-frame unit $\left[C_{\mathrm{u}}^{\mathrm{s}}\right]$, it is assumed that supplemental viscous dampers are installed between two adjacent floors throughout for the MS control purpose. Since the supplemental damping is much larger than the structural damping, the latter is neglected in the analysis. The global damping matrix of the whole subframe $\left[C^{s}\right]$ is formed by assembling all the unit damping matrices.

The mass matrix of the mega-sub-building is:

$$
\left[\boldsymbol{M}^{\mathrm{ms}}\right]=\left[\begin{array}{cc}
{\left[\boldsymbol{M}^{\mathrm{m}}\right]} & {[0]} \\
{[0]} & {\left[\boldsymbol{M}^{\mathrm{s}}\right]}
\end{array}\right]
$$

where $\left[M^{\mathrm{s}}\right]=$ diagonal matrix consisting of all the sub-masses.
The stiffness matrix of the mega-sub-building becomes:

$$
\left[K^{\mathrm{ms}}\right]=\left[K^{s}\right]+\left[\begin{array}{cc}
{\left[K^{\mathrm{m}}\right]} & {[0]} \\
{[0]} & {[0]}
\end{array}\right]
$$

Similarly, the damping matrix of the mega-sub-building is expressed as:

$$
\left[C^{\mathrm{ms}}\right]=\left[C^{\mathrm{s}}\right]+\left[\begin{array}{cc}
{\left[C^{\mathrm{m}}\right]} & {[0]} \\
{[0]} & {[0]}
\end{array}\right]
$$

Finally, the equation of motion for the mega-subbuilding is:

$$
\left[M^{\mathrm{ms}}\right]\left\{\ddot{X}^{\mathrm{ms}}\right\}+\left[C^{\mathrm{ms}}\right]\left\{\dot{X}^{\mathrm{ms}}\right\}+\left[K^{\mathrm{ms}}\right]\left\{X^{\mathrm{ms}}\right\}=\left\{F^{\mathrm{ms}}\right\}
$$

where $\left\{X^{\mathrm{ms}}\right\}=$ deformation vector of the mega-subbuilding,

$$
\left\{F^{\mathrm{ms}}\right\}=\left\{\begin{array}{c}
\left\{F^{\mathrm{m}}\right\} \\
\{0\}
\end{array}\right\}
$$

and $\left\{F^{\mathrm{m}}\right\}=$ wind force vector.

For an isolated mega-sub-controlled building shown in Fig. 1(c), a sub-structure can be modeled as a rigid mass, since the stiffness of the sub-structure is much larger than that of the isolators. Figure 3 shows the analytical model of the isolated mega-sub-controlled building.

\section{WIND LOADS}

Through the last few decades, extensive studies have been conducted to develop a wind model which can represent the characteristics of wind gust by a random process. Among them the Davenport model, considering both temporal and spatial variations, is one of the most accepted models and is used in this study. Based on the PSD of gusty wind, and with the help of fast Fourier transformation (FFT), the time histories of the lift and drag forces acting on the building are generated for the
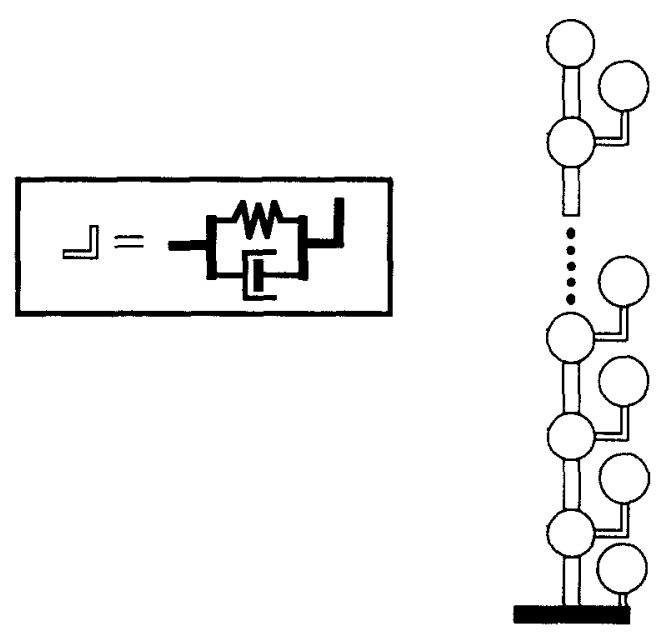

Fig. 3. Analytical model of isolated mega-sub-building. 
numerical simulation of the building response. In doing so, the cross-spectral density function of the wind loads along the height of the building is derived in order to obtain the MSV of building responses by the random vibration theory.

\section{Drag and lift forces}

The wind speed profile along the building height is expressed as:

$$
U(z, t)=\bar{U}(z)+u(z, t)
$$

where $\bar{U}(z)=$ mean wind speed at height $z$ and $u(z, t)=$ fluctuating wind speed with a specified non-white spectrum.

The drag force in the along-wind $(x)$ direction can be expressed as: ${ }^{3,5,6}$

$$
\begin{aligned}
f_{x, i}(t)= & \frac{1}{2} \rho C_{\mathrm{D}} A_{i}\left(U\left(z_{i}, t\right)-\dot{w}_{x, i}^{\mathrm{m}}\right)^{2} \\
& +\frac{\pi}{4} \rho C_{\mathrm{M}} A_{i} b\left(\dot{U}\left(z_{i}, t\right)-\ddot{w}_{x, i}^{\mathrm{m}}\right)
\end{aligned}
$$

where $\rho=$ air density, $b=$ lateral dimension of the building in the along-wind direction, $A_{i}=$ tributary area of the building at location $i$ in the along-wind direction, $C_{\mathrm{D}}=$ drag force coefficient, $C_{\mathrm{M}}=$ coefficient of virtual mass, and $w_{x, i}^{\mathrm{m}}=$ deflection of the building at location $i$ in the along-wind direction.

In this equation, the interaction between the wind and the building has been taken into account. The second term of the equation accounts for the net force resulting from the accelerated air mass around the body which is generally believed to be small compared with the first term and, thus, can be neglected in the numerical computation, as advocated by Simiu and Scanlan. ${ }^{4}$

The lift force acting on the building in the across-wind (y) direction is induced by the oscillation resulting from the vortex shedding. The lift force depends on both the shedding frequency and the building response, ${ }^{7,8}$ and it is modeled as: ${ }^{9}$

$$
\begin{aligned}
f_{y, i}(t)= & \frac{1}{2} \rho A_{i}^{\prime}\left(U\left(z_{i}, t\right)-\dot{w}_{x, i}^{\mathrm{m}}\right)^{2}\left[Y_{1}(\bar{\omega}) \frac{\dot{w}_{y, i}^{\mathrm{m}}}{\left(U\left(z_{i}, t\right)-\dot{w}_{x, i}^{\mathrm{m}}\right)}\right. \\
& +Y_{2}(\bar{\omega}) \frac{\left(w_{y, i}^{\mathrm{m}}\right)^{2} \dot{w}_{y, i}^{\mathrm{m}}}{D^{2}\left(U\left(z_{i}, t\right)-\dot{w}_{x, i}^{\mathrm{m}}\right)}-J_{1}(\bar{\omega}) \frac{w_{y, i}^{\mathrm{m}}}{D} \\
& \left.+J_{2}(\bar{\omega}) \frac{w_{y, i}^{\mathrm{m}}}{D} \sin \left(2 \omega_{\mathrm{s}} t\right)\right]
\end{aligned}
$$

where $A_{i}^{\prime}=$ tributary area of the building at location $i$ in the across-wind direction, $w_{y, i}^{\mathrm{m}}=$ deflection of the building at location $i$ in the across-wind direction, $\bar{\omega}=\omega_{1} D / \bar{U}(z)=$ reduced frequency, $\omega_{1}=$ fundamental frequency of the building, $\omega_{\mathrm{s}}=2 \pi \times \vartheta \bar{U}(z) / D=$ vortex shedding frequency, $\vartheta=$ Strouhal number, and $D=$ effective dimension of the building.

The four terms in eqn (8) respectively represent the linear and non-linear aeroelastic damping, aeroelastic stiffness, and parametric stiffness caused by vortex shedding. Goswami et al. ${ }^{8}$ verified this model and obtained the values of the coefficients $Y_{1}, Y_{2}, J_{1}$ and $J_{2}$ by performing a wind tunnel experiment with a circular cylinder.

\section{Fluctuating wind speed}

The time history of the fluctuating component of wind speed along the building height can be obtained with the aid of the two-dimensional spectral representation of stochastic processes and fields as established by Shinozuka and Deodatis ${ }^{10}$ as follows:

$$
\begin{aligned}
u(z, t)= & \sqrt{2} \sum_{i=1}^{N_{1}} \sum_{j=1}^{N_{2}} \sqrt{2 S\left(\omega_{i}, \kappa_{j}\right) \Delta \omega \Delta \kappa} \\
& \times\left[\cos \left(\omega_{i} t+\kappa_{j} z+\theta_{i j}\right)\right. \\
& \left.+\cos \left(\omega_{i} t-\kappa_{j} z+\phi_{i j}\right)\right]
\end{aligned}
$$

where $S\left(\omega_{i}, \kappa_{j}\right)=$ spectral density function of turbulent wind, $\omega_{i}=$ frequency, $\kappa_{j}=$ wave-number, $\Delta \omega=\omega_{\mathbf{u}} / N_{1}$, $\Delta \kappa=\kappa_{\mathrm{u}} / N_{2}, \omega_{\mathrm{u}}=$ upper cut-off frequency, $\kappa_{u}=$ upper cut-off wave number, $N_{1}=$ number of points in frequency axis, $N_{2}=$ number of points in wave number axis, and $\phi_{i j}, \phi_{i j}=$ independent random phase angles, uniformly distributed between 0 and $2 \pi$.

The following two-sided PSD function of the turbulent wind was suggested by Davenport ${ }^{10,11}$ and used by Vaicaitis et al. for the application in the spectral representation method:

$$
S(\omega, \kappa)=\frac{K^{\prime} \Phi^{2}}{2 \pi^{2}} \frac{|\omega|}{\left[1+\left(\frac{\Phi \omega}{2 \pi \tilde{U}(10)}\right)^{2}\right]^{4 / 3}} \frac{\epsilon|\omega|}{\pi\left(\epsilon^{2} \omega^{2}+\kappa^{2}\right)}
$$

where $\Phi=$ scale of turbulence, $K^{\prime}=$ surface drag coefficient, $\epsilon=$ constant $\left[\approx 0.006\left(\mathrm{~m} \mathrm{~s}^{-1}\right)\right]$ and $\bar{U}(10)=$ mean wind speed at $10 \mathrm{~m}$ height above the ground.

The distribution of mean wind speed follows the logarithmic law as below:

$$
U(z)=\bar{U}(10) \frac{\ln \left(z / z_{0}\right)}{\ln \left(10 / z_{0}\right)}
$$

where $z_{0}=$ roughness length.

Figures 4-6 show the plot of the PSD expressed in eqn (10), time history of fluctuating wind speed at the altitude of $188 \mathrm{~m}$ and the fluctuating wind speed along the height of building at the time instant of one second, respectively. The upper cut-off frequency, upper cut-off wave-number and roughness length are $10 \mathrm{~Hz}, 0.08 \mathrm{~m}^{-1}$ and $0.07 \mathrm{~m}$, respectively. The former two values are chosen according to the PSD plot of fluctuating wind and the last value decided under the assumption that the building is located in a suburban area. The following coefficient and parameter values are also used: $K^{\prime}=0.03, \Phi=1200 \mathrm{~m}$, and $U(10)=21 \mathrm{~m} \mathrm{~s}^{-1}$. It 


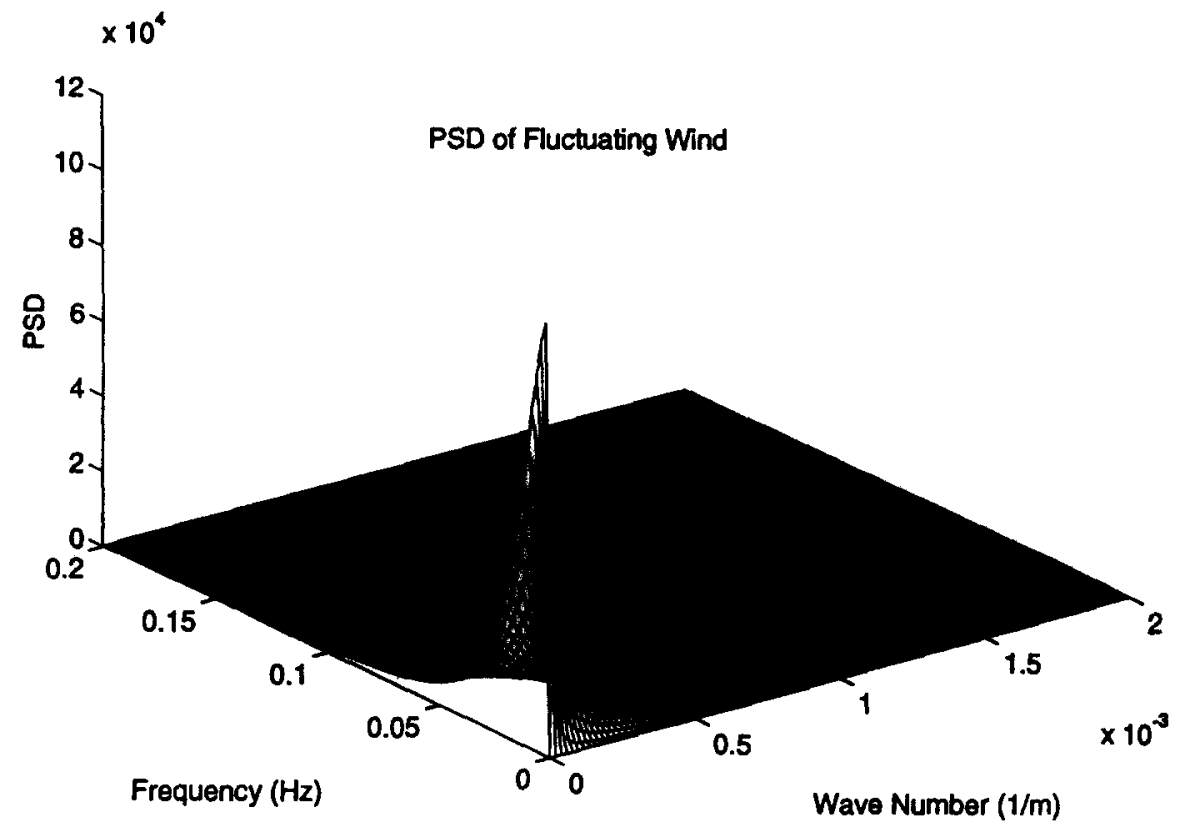

Fig. 4. Power spectral density of fluctuating wind speed.

Fluctuating Wind Speed at $188 \mathrm{~m}$

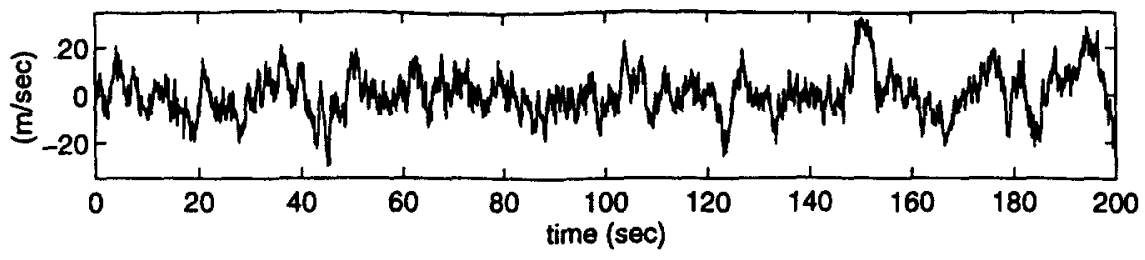

Fig. 5. Time history of fluctuating wind speed.

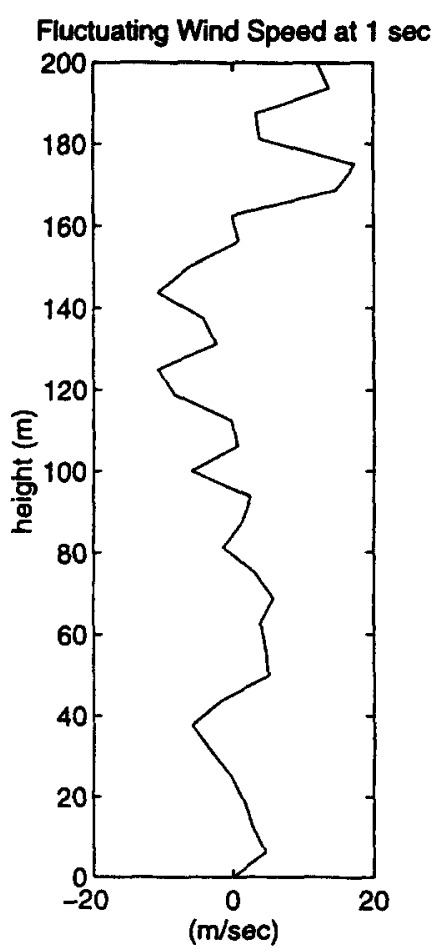

Fig. 6. Spatial variation of fluctuating wind speed. is noted that the FFT algorithm is only applied to one dimension (frequency) to calculate the fluctuating wind speed from eqn (9). Therefore, the frequency axis is equally divided by $N_{1}=16384$. However, the wave number axis is divided into three sections. The first section, between $\kappa=0$ and $0.008 \mathrm{~m}^{-1}$, is divided by $N_{2}=500$; the second, between $\kappa=0.008$ and $0.04 \mathrm{~m}^{-1}$ by $N_{2}=300$; and the third, between $\kappa=0.04$ and $0.08 \mathrm{~m}^{-1}$ by $N_{2}=100$. The section with higher magnitude is divided into smaller intervals to sufficiently represent the power of the density function. It is observed from Figs 5 and 6 that the spatial variation of the fluctuating wind is not as pronounced as the temporal variation, which is expected because the power of spectral density is concentrated in small wave number range, as shown in Fig. 4. The PSD value at $\omega=\kappa=0$ is $K^{\prime} \Phi^{2} /(2 \pi \epsilon)$ by first letting $\kappa$ approach zero and then $\omega$.

The standard deviation (STD) of fluctuating wind speed $\sigma_{\mathrm{u}}$ is calculated by the following equation with the knowledge that its mean value is equal to zero:

$$
\sigma_{\mathrm{u}}^{2}=4 \int_{0}^{\omega_{\mathrm{u}}} \int_{0}^{\kappa_{\mathrm{u}}} S(\omega, \kappa) \mathrm{d} \kappa \mathrm{d} \omega
$$

In order to verify the accuracy of numerical 
simulation, the average STD of 20 time histories generated from eqn (9), which is $8.6440 \mathrm{~m} \mathrm{~s}^{-1}$, is compared with the one obtained by eqn (12), which is $8.6694 \mathrm{~m} \mathrm{~s}^{-1}$. Excellent agreement between the two approaches has been demonstrated.

\section{Linearization of drag force}

The lift force expressed in eqn (8) involves highly nonlinear terms caused by the interaction between the building and wind, making it extremely difficult to apply the random vibration theory to analyze the effect of the lift force on the building response. The drag force given in eqn (7), however, can be linearized with several reasonable assumptions. By doing so, the random vibration theory is applicable to the analysis of the drag force.

Neglecting the acceleration term of the drag force in eqn (7) and then substituting eqn (6) into eqn (7), the wind force in the along-wind direction becomes

$$
\begin{aligned}
f_{x, i}(t)= & \frac{1}{2} \rho C_{\mathrm{D}} A_{i}\left(\bar{U}\left(z_{i}\right)^{2}+2 \bar{U}\left(z_{i}\right) u\left(t, z_{i}\right)-2 \bar{U}\left(z_{i}\right) \dot{w}_{x, i}^{\mathrm{m}}\right. \\
& \left.-2 u\left(t, z_{i}\right) \dot{w}_{x, i}^{\mathrm{m}}+u\left(t, z_{i}\right)^{2}+\dot{w}_{x, i}^{m^{2}}\right)
\end{aligned}
$$

The first term in eqn (13) represents the static force which will not affect the dynamic behavior of the building, and is thus ignored in the present analysis. Compared with the mean wind velocity, the velocity response of the mega-structure and the velocity of fluctuating wind are small. Therefore, the last three higher order terms of the right hand side of eqn (13) can be neglected. The validity of this assumption will be verified in the numerical example described later. Finally, the effective drag force is defined as the following equation, by ignoring the terms in the right hand side of eqn (13) which do not significantly affect the building's dynamic behavior:

$$
f_{x, i}^{\mathrm{e}}(t)=\rho C_{\mathrm{D}} A_{i} \bar{U}\left(z_{i}\right)\left(u\left(t, z_{i}\right)-\dot{w}_{x, i}^{\mathrm{m}}\right)
$$

The second term of eqn (14) can be moved to the left side of the equation of motion as an additional damping term; therefore, the effective force in the equation of motion for a continuous structural model is:

$$
f_{i}(t)=\rho C_{\mathrm{D}} b \bar{U}\left(z_{i}\right) u\left(t, z_{i}\right) \mathrm{d} z=c\left(z_{i}\right) u\left(t, z_{i}\right) \mathrm{d} z
$$

With a lumped-mass structural model, the effective force at the $j$ th building mass may be expressed in approximation as:

$$
F_{j}(t)=\int_{z_{j 1}}^{z_{j 2}} c\left(z_{j}\right) u(t, z) \mathrm{d} z
$$

where $z_{j 1}=$ height of the lower boundary of the tributary area represented by mass $j$, and $z_{j 2}=$ height of the upper boundary of the tributary area represented by mass $j$.

Two assumptions have been made in order to derive eqn (16) from eqn (14). Firstly, the mean wind speed at locations from $z_{j 1}$ to $z_{j 2}$ is represented by that at mass $j$, which is acceptable because the spatial variation of mean wind speed is relatively smaller at high attitudes where the effective force contributes more substantially to the building response. Secondly, the building's velocity response at mass $j$ also represents that at other locations among the tributary area, because that is the location where the responses are evaluated.

\section{Cross-spectral density function of effective force}

In this study, the effective forces are used for the optimal design of MS buildings. To accomplish this, it is necessary to calculate the cross-spectral density functions of input effective force in order to obtain the PSD of building response.

The cross-correlation function of the effective force at mass $i$ and $j$ is

$$
R_{F_{i} F_{j}}(\tau)=E\left[F_{i}(t) F_{j}(t+\tau)\right]
$$

Substituting eqn (16) into eqn (17)

$$
\begin{aligned}
R_{F_{i} F_{j}}(\tau)= & E\left[\left[\int_{z_{i 1}}^{z_{i 2}} c\left(z_{i}\right) u(t, \alpha) \mathrm{d} \alpha\right]\right. \\
& \left.\times\left[\int_{z_{j 1}}^{z_{j 2}} c\left(z_{j}\right) u(t+\tau, \beta) \mathrm{d} \beta\right]\right] \\
= & \int_{z_{i 1}}^{z_{i 2}} c\left(z_{i}\right)\left\{\int_{z_{j 1}}^{z_{j 2}} c\left(z_{j}\right) E[u(t, \alpha) u(t+\tau, \beta)] \mathrm{d} \beta\right\} \mathrm{d} \alpha \\
= & \int_{z_{i 1}}^{z_{i 2}} c\left(z_{i}\right)\left\{\int_{z_{j 1}}^{z_{j 2}} c\left(z_{j}\right)\left[R_{u u}(\tau, \beta-\alpha)\right] \mathrm{d} \beta\right\} \mathrm{d} \alpha
\end{aligned}
$$

The auto-correlation function of the fluctuating wind speed $R_{u u}(\tau, \beta-\alpha)$ is:

$$
R_{u u}(\tau, \beta-\alpha)=\int_{-\infty}^{\infty}\left[\int_{-\infty}^{\infty} S(\omega, \kappa) \mathrm{e}^{i \kappa(\beta-\alpha)} \mathrm{d} \kappa\right] \mathrm{e}^{i \omega \tau} \mathrm{d} \omega
$$

where $S(\omega, \kappa)$ is the PSD function of the fluctuating wind speed in eqn (10).

$R_{u u}(\tau, \beta-\alpha)$ may also be obtained through WienerKhinchin transformation:

$$
R_{u u}(\tau, \beta-\alpha)=\int_{-\infty}^{\infty} S^{\prime}(\omega, \beta-\alpha) \mathrm{e}^{i \omega \tau} \mathrm{d} \omega
$$

Comparing eqn (19) with eqn (20):

$$
S^{\prime}(\omega, \beta-\alpha)=\int_{-\infty}^{\infty} S(\omega, \kappa) \mathrm{e}^{i \kappa(\beta-\alpha)} \mathrm{d} \kappa
$$

The cross-spectral density function of effective force at mass $i$ and $j$ is then obtained by:

$$
S_{F_{i} F_{j}}(\omega)=\frac{1}{2 \pi} \int_{-\infty}^{\infty} R_{F_{i} F_{j}}(\tau) \mathrm{e}^{-i \omega \tau} \mathrm{d} \tau
$$


Substituting eqn (18) into eqn (22):

$$
\begin{aligned}
S_{F_{i} F_{j}}(\omega)= & \int_{z_{i 1}}^{z_{i 2}} c\left(z_{i}\right)\left\{\int_{z_{j 1}}^{z_{j 2}} c\left(z_{j}\right)\right. \\
& \left.\times\left[\frac{1}{2 \pi} \int_{-\infty}^{\infty} R_{u u}(\tau, \beta-\alpha) \mathrm{e}^{-i \omega \tau} \mathrm{d} \tau\right] \mathrm{d} \beta\right\} \mathrm{d} \alpha \\
= & \int_{z_{i 1}}^{z_{i 2}} c\left(z_{i}\right)\left\{\int_{z_{j 1}}^{z_{j 2}} c\left(z_{j}\right)\left[S^{\prime}(\omega, \beta-\alpha)\right] \mathrm{d} \beta\right] \mathrm{d} \alpha
\end{aligned}
$$

Substituting eqn (21) into eqn (23),

$$
\begin{aligned}
S_{F_{i} F_{j}}(\omega)= & \int_{z_{i 1}}^{z_{i 2}} c\left(z_{i}\right)\left\{\int_{z_{j 1}}^{z_{j 2}} c\left(z_{j}\right)\right. \\
& \left.\times\left[\int_{-\infty}^{\infty} S(\omega, \kappa) \mathrm{e}^{i \kappa(\beta-\alpha)} \mathrm{d} \kappa\right] \mathrm{d} \beta\right\} \mathrm{d} \alpha
\end{aligned}
$$

Finally, substituting eqn (10) into eqn (24),

$$
\begin{aligned}
& S_{F_{i} F_{j}}(\omega)= \int_{z_{i 1}}^{z_{i 2}} c\left(z_{i}\right)\left\{\int_{z_{j 1}}^{z_{j 2}} c\left(z_{j}\right)\right. \\
& \times\left[\int_{-\infty}^{\infty}\left[B(\omega) \frac{1}{(\epsilon \omega)^{2}+\kappa^{2}}\right]\right. \\
&\left.\left.\times \mathrm{e}^{i \kappa(\beta-\alpha)} \mathrm{d} \kappa\right] \mathrm{d} \beta\right\} \mathrm{d} \alpha \\
&= c\left(z_{i}\right) c\left(z_{j}\right) B(\omega) \int_{-\infty}^{\infty} \frac{1}{(\epsilon \omega)^{2}+\kappa^{2}} \\
& \times\left\{\int_{z_{i 1}}^{z_{i 2}}\left[\int_{z_{j 1}}^{z_{j 2}} \mathrm{e}^{i \kappa \beta} \mathrm{d} \beta\right] \mathrm{e}^{-i \kappa \alpha} \mathrm{d} \alpha\right\} \mathrm{d} \kappa \\
&= c\left(z_{i}\right) c\left(z_{j}\right) B(\omega) \int_{-\infty}^{\infty} \frac{1}{(\epsilon \omega)^{2}+\kappa^{2}} \\
& \times\left\{\int_{z_{i 1}}^{z_{i 2}} \frac{1}{i \kappa}\left(\mathrm{e}^{i \kappa z_{j 2}}-\mathrm{e}^{i \kappa z_{j 1}}\right) \mathrm{e}^{-i \kappa \alpha} \mathrm{d} \alpha\right\} \mathrm{d} \kappa \\
&= c\left(z_{i}\right) c\left(z_{j}\right) B(\omega) \int_{-\infty}^{\infty} \frac{1}{(\epsilon \omega)^{2}+\kappa^{2}} \\
& \times\left\{\frac{1}{\kappa^{2}}\left(\mathrm{e}^{i \kappa z_{j 2}}-\mathrm{e}^{i \kappa z_{j 1}}\right)\left(\mathrm{e}^{-i \kappa z_{i 1}}-\mathrm{e}^{-i \kappa z_{i 2}}\right)\right\} \mathrm{d} \kappa \\
&= c\left(z_{i}\right) c\left(z_{j}\right) B(\omega) \\
& \times \int_{-\infty}^{\infty}\left\{\frac{1}{(\epsilon \omega)^{2}}\left(\frac{1}{\kappa^{2}}-\frac{1}{(\epsilon \omega)^{2}+\kappa^{2}}\right)\right. \\
& \times\left[\mathrm{e}^{i \kappa\left(z_{j 2}-z_{i 1}\right)}+\mathrm{e}^{i \kappa\left(z_{j 1}-z_{i 2}\right)}\right. \\
&\left.\left.-\mathrm{e}^{i \kappa\left(z_{j 2}-z_{i 2}\right)}-\mathrm{e}^{i \kappa\left(z_{j 1}-z_{i 1}\right)}\right]\right\} \mathrm{d} \kappa \\
&
\end{aligned}
$$

Since

$$
\int_{-\infty}^{\infty} \frac{1}{x^{n}} \mathrm{e}^{i a x} \mathrm{~d} x=\pi i \operatorname{sgn}(a) \frac{(i a)^{n-1}}{(n-1) !}
$$

and

$$
\int_{-\infty}^{\infty} \frac{1}{c^{2}+x^{2}} \mathrm{e}^{i a x} \mathrm{~d} x=\frac{\pi \mathrm{e}^{c|a|}}{c}
$$

$$
\begin{aligned}
S_{F_{i} F_{j}}(\omega)= & B(\omega)\left[\frac{\pi c\left(z_{i}\right) c\left(z_{j}\right)}{(\epsilon \omega)^{2}}\right]\left[\Gamma+\frac{\Delta}{\epsilon|\omega|}\right] \\
= & {\left[\frac{K^{\prime} \Phi^{2}}{2 \pi^{3}} \frac{\epsilon \omega^{2}}{\left[1+\left(\frac{\Phi \omega}{2 \pi \bar{U}(10)}\right)^{2}\right]^{4 / 3}}\right] } \\
& \times\left[\frac{\pi c\left(z_{i}\right) c\left(z_{j}\right)}{(\epsilon \omega)^{2}}\right]\left[\Gamma+\frac{\Delta}{\epsilon|\omega|}\right] \\
= & \frac{c\left(z_{i}\right) c\left(z_{j}\right)}{2 \pi^{2}} \frac{K^{\prime} \Phi^{2}}{\left[1+\left(\frac{\Phi \omega}{2 \pi \bar{U}(10)}\right)^{2}\right]^{4 / 3}} \\
& \times\left(\frac{\Gamma}{\epsilon}+\frac{\Delta}{\epsilon^{2}|\omega|}\right)
\end{aligned}
$$

where $\Gamma=\left|z_{j 2}-z_{i 1}\right|+\left|z_{j 1}-z_{i 2}\right|-\left|z_{j 2}-z_{i 2}\right|-\left|z_{j 1}-z_{i 1}\right|$

$$
\begin{aligned}
\Delta= & \mathrm{e}^{-\epsilon|\omega|\left|z_{j 2}-z_{i 1}\right|}+\mathrm{e}^{-\epsilon|\omega|\left|z_{j 1}-z_{i_{2}}\right|} \\
& -\mathrm{e}^{-\epsilon|\omega|\left|z_{j 2}-z_{i 2}\right|}-\mathrm{e}^{-\epsilon|\omega|\left|z_{j 1}-z_{i_{1}}\right|}
\end{aligned}
$$

Equation (25) will be used to calculate the PSD functions of the building response for the frequency domain analysis in order to optimally design the dynamic characteristics of sub-structures, as shown in the numerical example given later.

Assuming that the fluctuating wind speed at mass $j$ represents that at other locations along the tributary area, the effective force in eqn (16) becomes:

$$
F_{j}(t)=c\left(z_{j}\right)\left(z_{j 2}-z_{j 1}\right) u\left(t, z_{j}\right)
$$

and eqn (24) becomes

$$
\begin{aligned}
S_{F_{i} F_{j}}(\omega)= & c\left(z_{i}\right) c\left(z_{j}\right)\left(z_{i 2}-z_{i 1}\right)\left(z_{j 2}-z_{j 1}\right) \\
& \times \int_{-\infty}^{\infty} S(\omega, \kappa) \mathrm{e}^{i \kappa\left(z_{j}-z_{i}\right)} \mathrm{d} \kappa
\end{aligned}
$$

where $z_{j}=$ the height of mass $j$.

Therefore, the cross-spectral density function in eqn (25) becomes

$$
\begin{aligned}
S_{F_{i} F_{j}}(\omega)= & \frac{c\left(z_{i}\right) c\left(z_{j}\right)\left(z_{i 2}-z_{i 1}\right)\left(z_{j 2}-z_{j 1}\right)}{2 \pi^{2}} \\
& \times \frac{K^{\prime} \Phi^{2}|\omega|}{\left[1+\left(\frac{\Phi \omega}{2 \pi \bar{U}(10)}\right)^{2}\right]^{4 / 3}} \mathrm{e}^{-\varepsilon|\omega|\left|z_{j}-z_{i}\right|}
\end{aligned}
$$

The cross-spectral density function in eqn (28) is an approximation of that shown in eqn (25), under the assumption that the fluctuating wind speed acting at mass $j$ represents that at other locations along the tributary area. This assumption will be used to generate time histories of wind force for numerical simulation in time domain. The comparison between the plots of eqns (25) and (28) can verify the validity of this assumption. It will be conducted in the numerical example later. 


\section{DESIGN OF OPTIMAL DYNAMIC PARAMETERS}

The dynamic parameters of the mega-sub-controlled building, including the stiffness and damping ratios of the sub-structures, are designed to minimize certain critical responses (referred to here as target responses) of the mega-sub-building under the stochastic wind loads, such as (1) the response acceleration of the sub-structure for the purpose of improving human comfort and protecting sensitive contents, and (2) the deformation of the mega-structure for the structural safety purpose. In this study, the displacement of the bottom first megafloor and the acceleration of the top sub-floor are chosen to be the target responses, for the reasons that the base shear of the mega-structure is the primary parameter for structural safety evaluation and the response acceleration of the sub-structure at the top location is usually larger than those at other locations (see the numerical simulation result given in Table 1).

The MSV of the target responses, obtained through the random vibration theory, serve as performance indices for optimization. They are calculated from the PSD functions of the target responses which are derived from the frequency response functions of the mega-subbuilding and the power- and cross-spectral density functions of effective wind force.

\section{PSD, MSV and STD of building response}

Rewriting the equations of motion for the mega-subbuilding given in eqn (5) in a state-space form:

$$
\begin{aligned}
& \dot{\mathbf{X}}(t)=\mathbf{A X}(t)+\mathbf{B F}(t) \\
& \mathbf{Y}(t)=\mathbf{C X}(t)+\mathbf{D F}(t)
\end{aligned}
$$

where $\mathbf{X}(t)=$ state vector, $\mathbf{Y}(t)=$ output vector consisting of the target responses, $\mathbf{F}(t)=$ wind force vector in the along-wind direction, and $\mathbf{A}, \mathbf{B}, \mathbf{C}, \mathbf{D}=$ corresponding matrices derived from eqn (5).

For the purpose of optimally designing the dynamic parameters of a mega-sub-controlled building, the drag force in eqn (14), which excludes the static and higher order terms, is used. Therefore, the wind force vector in the along-wind direction becomes:

$$
\mathbf{F}(t)=F_{j}(t)-P X(t)
$$

Table 1. Peak building response

\begin{tabular}{lrlr}
\hline $\begin{array}{l}\text { (a) Mega-sub controlled } \\
\text { building }\end{array}$ & \multicolumn{2}{c}{$\begin{array}{c}\text { (b) Isolated mega-sub } \\
\text { controlled building }\end{array}$} \\
\hline$x_{m 1}(\mathrm{~cm})$ & 2.5215 & $x_{m 1}(\mathrm{~cm})$ & $2 \cdot 5341$ \\
$x_{m 2}-x_{m 1}(\mathrm{~cm})$ & 5.5947 & $x_{m 2}-x_{m 1}(\mathrm{~cm})$ & $5 \cdot 7409$ \\
$x_{m 3}-x_{m 2}(\mathrm{~cm})$ & 6.6578 & $x_{m 3}-x_{m 2}(\mathrm{~cm})$ & 6.8578 \\
$\ddot{x}_{s 1}(\mathrm{gal})$ & 5.4414 & $\ddot{x}_{s 1}(\mathrm{gal})$ & 6.3881 \\
$\ddot{x}_{s 2}(\mathrm{gal})$ & 8.0595 & $\ddot{x}_{s 2}(\mathrm{gal})$ & $20 \cdot 7431$ \\
$\ddot{x}_{s 3}(\mathrm{gal})$ & 8.6338 & & \\
$\ddot{x}_{s 4}(\mathrm{gal})$ & 11.5155 & & \\
$\ddot{x}_{s 5}$ (gal) & 18.0802 & & \\
$\ddot{x}_{s 6}$ (gal) & 15.4875 & & \\
\hline
\end{tabular}

where $\mathbf{F}_{j}(t)=$ effective force vector with its elements calculated by eqn (16):

$\mathbf{P}=$

$\left[[0]_{n \times(n+p)}\left[\begin{array}{ccc}\left.c\left(z_{1}\right) z_{12}-z_{11}\right) \bar{U}\left(z_{1}\right) & & 0 \\ 0 & \ddots & c\left(z_{n}\right)\left(z_{n 2}-z_{n 1}\right) \breve{U}\left(z_{n}\right)\end{array}\right]_{n \times n}[0]_{n \times p}\right]_{n \times 2(n+p)}$

$=$ constant matrix.

Substituting eqn (30) into eqn (29):

$$
\begin{aligned}
& \dot{\mathbf{X}}(t)=[\mathbf{A}-\mathbf{B P}] \mathbf{X}(t)+\mathbf{B} \mathbf{F}_{j}(t)=\overline{\mathbf{A}} \mathbf{X}(t)+\mathbf{B} \mathbf{F}_{j}(t) \\
& \mathbf{Y}(t)=[\mathbf{C}-\mathbf{D P}] \mathbf{X}(t)+\mathbf{D} \mathbf{F}_{j}(t)=\overline{\mathbf{C}} \mathbf{X}(t)+\mathbf{D F} \mathbf{F}_{j}(t)
\end{aligned}
$$

The frequency response function matrix, $\left[H_{i j}(\omega)\right]$, of the output vector $\mathbf{Y}(t)$ is obtained:

$$
\left[H_{i j}(\omega)\right]=\overline{\mathbf{C}}[i \omega \mathbf{I}-\overline{\mathbf{A}}]^{-1} \mathbf{B}+\mathbf{D}
$$

where $\mathbf{I}=$ identity matrix.

For a multi-input single-output system, the $i$ th response can be expressed as:

$$
y_{i}(t)=\sum_{j=1}^{n} \int_{-\infty}^{\infty} F_{j}(t-\delta) h_{i j}(\delta) \mathrm{d} \delta
$$

where $h_{i j}(\tau)=$ impulse transfer function from input $j$ to response $i$.

The auto-correlation function of $y_{i}(t)$ is derived as:

$$
\begin{aligned}
R_{y_{i} y_{i}}(\tau)= & E[y(t) y(t+\tau)] \\
= & E\left[\left[\sum_{j=1}^{n} \int_{-\infty}^{\infty} \mathbf{F}_{j}(t-\delta) h_{i j}(\delta) \mathrm{d} \delta\right]\right. \\
& \left.\times\left[\sum_{j=1}^{n} \int_{-\infty}^{\infty} \mathbf{F}_{j}(t+\tau-\lambda) h_{i j}(\lambda) \mathrm{d} \lambda\right]\right] \\
= & \int_{-\infty}^{\infty} \int_{-\infty}^{\infty}\left\{\sum _ { j = 1 } ^ { n } \sum _ { h = 1 } ^ { n } \left[E \left[\mathbf{F}_{j}(t-\delta)\right.\right.\right. \\
& \left.\left.\left.\times \mathbf{F}_{h}(t+\tau-\lambda)\right] h_{i j}(\delta) h_{i h}(\lambda)\right]\right\} \mathrm{d} \delta \mathrm{d} \lambda \\
= & \int_{-\infty}^{\infty} \int_{-\infty}^{\infty}\left\{\sum _ { j = 1 } ^ { n } \sum _ { h = 1 } ^ { n } \left[R_{\mathbf{F}_{j} \mathbf{F}_{h}}(\tau+\delta-\lambda)\right.\right. \\
& \left.\left.\times h_{i j}(\delta) h_{i h}(\lambda)\right]\right\} \mathrm{d} \delta \mathrm{d} \lambda
\end{aligned}
$$

Applying the Wiener-Khinchin transformation, the power spectral density function of response $y_{i}(t)$ is derived as:

$$
\begin{aligned}
S_{y_{i} y_{i}}(\omega)= & \frac{1}{2 \pi} \int_{-\infty}^{\infty} R_{y_{i} y_{i}}(\tau) \mathrm{e}^{-i \omega \tau} \mathrm{d} \tau \\
= & \frac{1}{2 \pi} \int_{-\infty}^{\infty}\left\{\int _ { - \infty } ^ { \infty } \int _ { - \infty } ^ { \infty } \left\{\sum_{j=1}^{n} \sum_{h=1}^{n}\right.\right. \\
& \left.\left.\times\left[R_{\mathbf{F}_{j} \mathbf{F}_{h}}(\tau+\delta-\lambda) h_{i j}(\delta) h_{i h}(\lambda)\right]\right\} \mathrm{d} \delta \mathrm{d} \lambda\right\} \\
& \times \mathrm{e}^{-i \omega \tau} \mathrm{d} \tau
\end{aligned}
$$




$$
\begin{aligned}
= & \sum_{j=1}^{n} \sum_{h=1}^{n}\left[\int_{-\infty}^{\infty} \frac{1}{2 \pi} R_{\mathbf{F}_{j} \mathbf{F}_{h}}(\tau+\delta-\lambda) \mathrm{e}^{-i \omega(\tau+\delta-\lambda)} \mathrm{d} \tau\right] \\
& \times\left[\int_{-\infty}^{\infty} h_{i j}(\delta) \mathrm{e}^{i \omega \delta} \mathrm{d} \delta\right]\left[\int_{-\infty}^{\infty} h_{i h}(\lambda) \mathrm{e}^{-i \omega \lambda} \mathrm{d} \lambda\right] \\
= & \sum_{j=1}^{n} \sum_{h=1}^{n} S_{\mathbf{F}_{j} \mathbf{F}_{h}}(\omega) H_{i j}^{*}(\omega) H_{i h}(\omega)
\end{aligned}
$$

where $S_{\mathbf{F}_{j} \mathbf{F}_{h}}(\omega)$ is the power- $(i=j)$ and cross-spectral $(i \neq j)$ density function of the effective force from eqn (25), and $H_{i j}(\omega)$ is the frequency response function from eqn (32); the star denotes the complex conjugate.

\section{Optimization}

The optimal values of the stiffness $E I_{\text {sopt }}$ (which is related to the optimal frequency $\omega_{\text {sopt }}$ ) and damping ratio $h_{\text {sopt }}$ of a sub-structure is obtained by minimizing the target responses in the along-wind direction, and the linearized drag force model in eqn (14) is used to represent the wind force. Then, the resulting optimal parameter values will be used to find the responses in the across-wind direction.

The MSV of the $i$ th target response is obtained by substituting its PSD calculated from eqn (35) into the following equation:

$$
E\left[y_{i}(t)^{2}\right]=R_{y_{i} y_{i}}(0)=2 \int_{0}^{\omega_{u}^{\prime}} S_{y_{i} y_{i}}(\omega) \mathrm{d} \omega
$$

where, $\omega_{u}^{\prime}=$ the cut-off frequency of $S_{y_{i} y_{i}}(\omega)$.

Since the mean response $E\left[y_{i}(t)\right]$ is equal to zero, the MSV of the response $E\left[y_{i}(t)^{2}\right]$ is equal to $\sigma_{y_{i}}^{2}$ where $\sigma_{y_{i}}$ is the standard deviation (STD) of response.

The STD of a target response $\sigma_{y_{i}}$ is a function of the dynamic parameters $\omega_{s}$ and $h_{s}$. Therefore, the optimal values $\omega_{\text {sopt }}$ and $h_{\text {sopt }}$ can be determined by minimizing the STD of a target building response numerically. This will be demonstrated by a numerical example in the next section.

\section{DEMONSTRATION WITH A NUMERICAL EXAMPLE}

\section{Analytical model}

An example mega-sub-building is presented to demonstrate the design procedure of a mega-sub-controlled building. The numerical simulation is conducted in the time domain to compare the performance of megasub-controlled buildings with different sub-structure configurations and to verify the STD of the target responses obtained through the random vibration theory in the frequency domain. The building is a $200 \mathrm{~m}$ high, cylindrical steel structure with a diameter of $40 \mathrm{~m}$. The mega-structure consists of three mega-floors and each sub-structure consists of three sub-floors. It is assumed that the damping ratios of the mega-structure for all the modes are equal to $1 \%$. The bottom substructure does not have a significant effect on the reduction of the target responses. ${ }^{13}$ In the analytical model, therefore, the weight of the bottom sub-structure is evenly distributed to the bottom mega-floor and ground. The analytical model and parameters of the mega-sub-controlled building are shown in Fig. 7(a). Another configuration of the mega-sub-controlled building is to base-isolate the sub-structures and its analytical model is shown in Fig. 7(b), with each substructure being modeled as a rigid mass.

\section{Optimization}

In order to determine the optimal values of the fundamental frequency and damping ratios of the substructures, the power- and cross-spectral density functions of the effective forces are calculated and shown in Figs 8 and 9. Figures 8(a) and 9(a) are calculated using eqn (25), while Figs 8(b) and 9(b) use eqn (28) which is an approximation of eqn (25) under the assumption that the fluctuating wind speeds at the whole tributary area
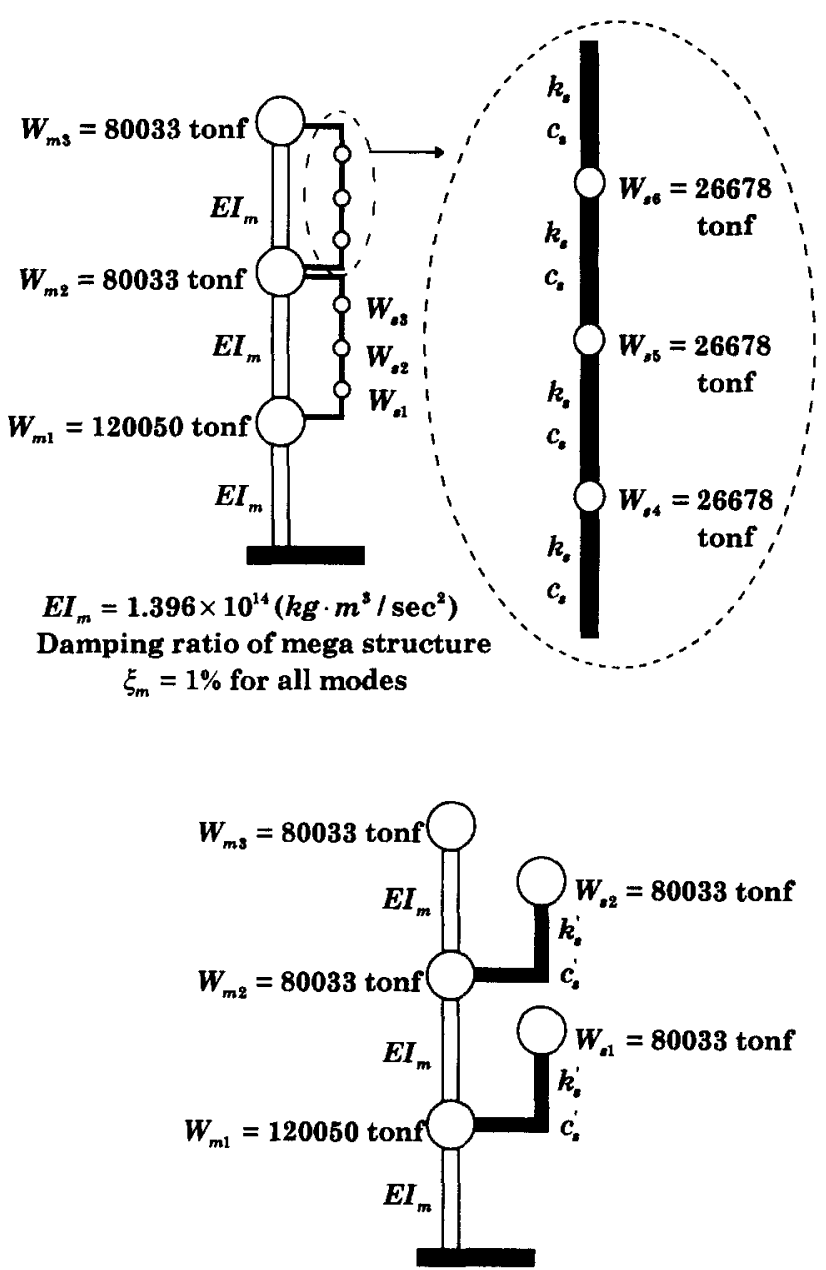

Fig. 7. Analytical model and parameters of example building. (a) Mega-sub-controlled building, (b) isolated model and parameters of example building. 

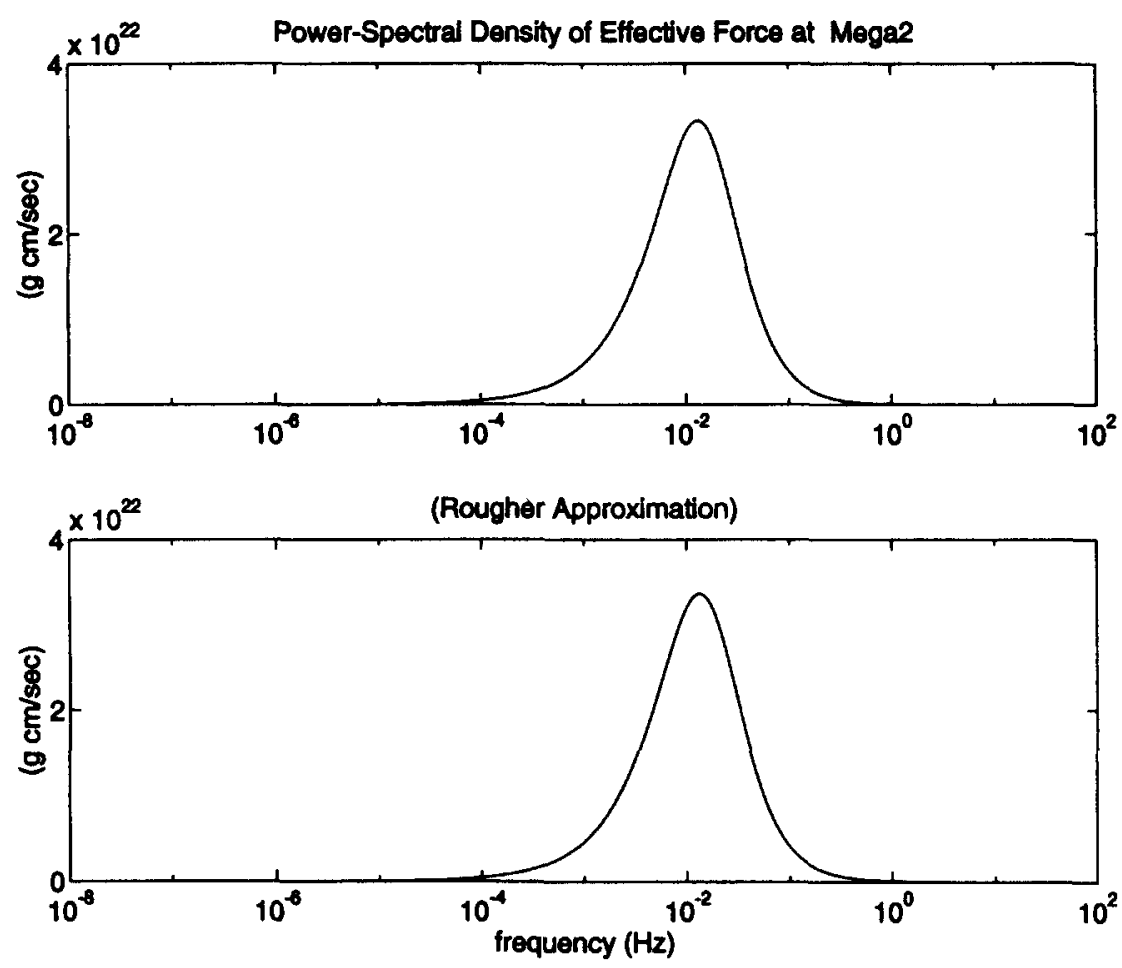

Fig. 8. Power-spectral density of effective force.

can be represented by the one at the middle level. No significant difference between Fig. 8(a) and (b) as well as between Fig. 9(a) and (b) is observed, which verifies the validity of the assumption. Therefore, in numerical simulation, the time histories of wind speed generated at all mega-mass locations are used to represent the wind speed at the whole tributary areas. However, the more accurate results from eqn (25) are used in the frequency domain analysis to calculate the PSD of the target responses.

As mentioned in the previous section, the optimal values of the dynamic parameters are obtained by numerically minimizing the STD of the target responses in the along-wind direction evaluated by means of the random vibration analysis method. The base shear coefficient of the mega-structure and the acceleration
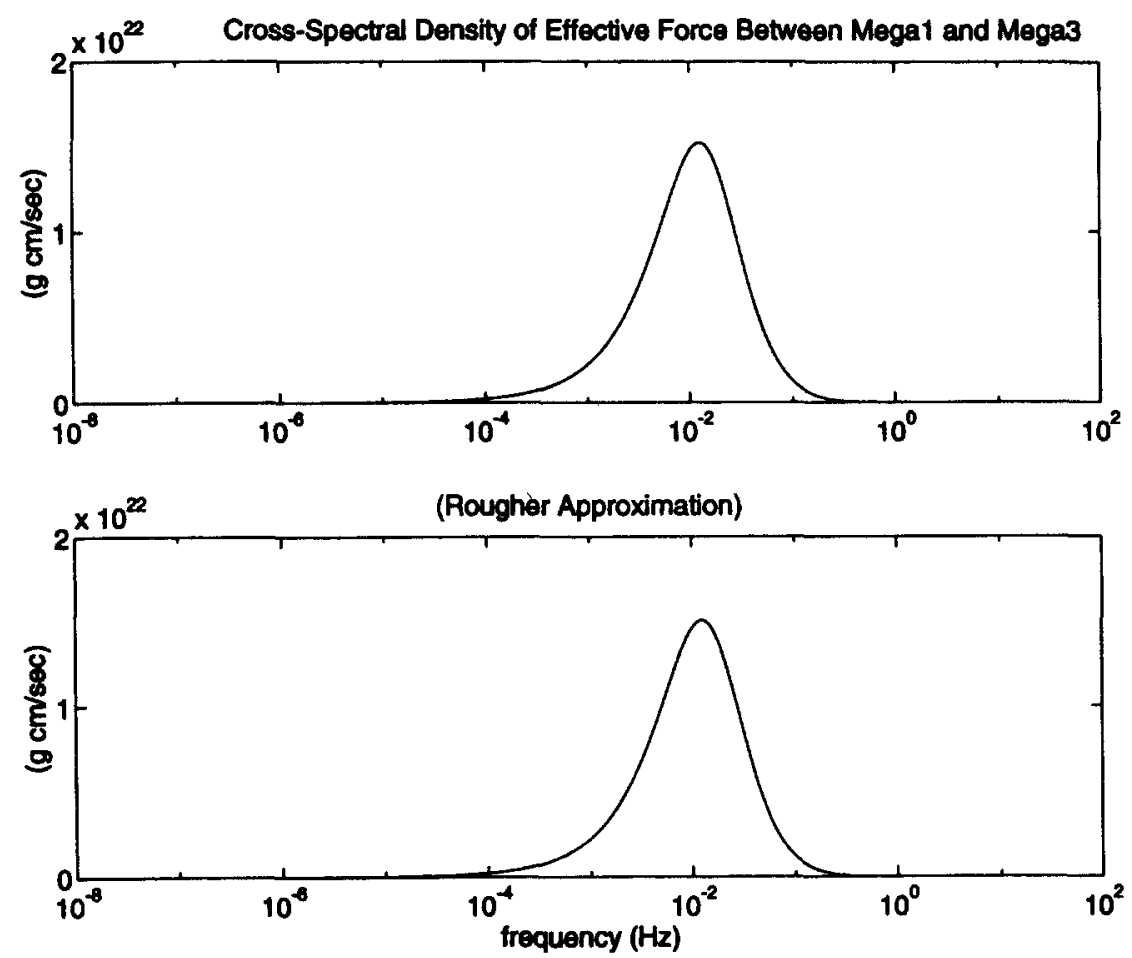

Fig. 9. Cross-spectral density of effective force 
of the top sub-floor are chosen to be the target responses. It is assumed that the mass, stiffness and damping of all the floors of the sub-structures are identical. The fundamental frequency $\omega_{\mathrm{s} 1}$, which is related to the stiffness, and the damping ratio $h_{\mathrm{s}}$ of the sub-structures become the dynamic parameters to be determined for the purpose of optimally controlling the building vibration under wind excitations. It is noted that $h_{\mathrm{s}}$ is defined as

$$
h_{\mathrm{s}}=\frac{c_{\mathrm{s}}}{2 m_{\mathrm{s}} \omega_{\mathrm{m}_{1}}}
$$

where $\omega_{\mathrm{m}_{1}}=$ fundamental frequency of the megastructure, $c_{\mathrm{s}}=$ damping coefficient of the sub-structure and $m_{\mathrm{s}}=$ mass of a sub-floor.

This definition makes $\omega_{\mathrm{s} 1}$ and $h_{\mathrm{s}}$ two independent parameters so that changing $\omega_{\mathrm{s} 1}$ with $h_{\mathrm{s}}$ fixed would not affect the damping coefficient $c_{\mathrm{s}}$. This is important for determining the optimal values of $\omega_{\mathrm{s} 1}$ and $h_{\mathrm{s}}$.

The STD of the target responses of the mega-subcontrolled building, the base shear coefficient of the mega-structure and the acceleration of the top sub-floor, are plotted in Fig. 10 as functions of $\omega_{\mathrm{s} 1}$ and $h_{\mathrm{s}}$. The values of $\omega_{\mathrm{s} 1}$ and $h_{\mathrm{s}}$, resulting in the minimum target responses, are considered to be the optimal values. As shown in the figure, the optimal values of $\omega_{\mathrm{s}}$ and $h_{\mathrm{s}}$ achieving the minimum base shear coefficient $\left(\omega_{\mathrm{s} 1}=\right.$ $0.2314 \mathrm{~Hz}$ and $\left.h_{\mathrm{s}}=0.3\right)$ are different from those achieving the minimum acceleration $\left(\omega_{\mathrm{s} 1}=0 \mathrm{~Hz}\right.$ and $h_{\mathrm{s}}=0$ ). Nevertheless, such a low fundamental frequency required for both purposes may be difficult to realize in the actual design of the sub-structures.
It is for this reason that a base-isolated megasub-building is suggested which can easily provide a low fundamental frequency for the sub-structures through isolation devices. The STD of the target responses for the isolated mega-sub-controlled building vs $\omega_{\mathrm{s} 1}$ and $h_{\mathrm{s}}$ are plotted in Fig. 11 and show similar shapes to those in Fig. 10. The minimum base shear coefficient of the mega-structure corresponds to the values of $\omega_{\mathrm{s} 1}=0.2785 \mathrm{~Hz}$ and $h_{\mathrm{s}}=0 \cdot 15$, while the minimum acceleration of the top sub-floor requires $\omega_{\mathrm{s} 1}=0 \mathrm{~Hz}$ and $h_{\mathrm{s}}=0$. By comparing the optimal values of $\omega_{\mathrm{s} 1}$, which can achieve the minimum base shear coefficient of the mega-structure, between the megasub- and isolated mega-sub-controlled buildings, it can be seen that both of them require a similar flexibility of the sub-structures which is more difficult to achieve by the former, but easier by the latter.

Figures 10 and 11 reveal a promising advantage of a mega-sub-controlled building. The control is robust because slight changes in the values of $\omega_{\mathrm{s} 1}$ and $h_{\mathrm{s}}$ will cause little change in the target responses of the building. Such robustness not only provides the structural designer with some freedom to select appropriate values of $\omega_{\mathrm{s} 1}$ and $h_{\mathrm{s}}$ which are more easily realizable, but also relieves the concern of performance deterioration caused by parameter changes due to construction and other factors.

\section{Numerical simulation}

Observing Figs 10 and 11 , it is noted that although the optimal values for minimizing the top sub-acceleration are found to be $\omega_{\mathrm{sl}}=0 \mathrm{~Hz}$ and $h_{\mathrm{s}}=0$, the optimal

\section{Base Shear Coefficient}

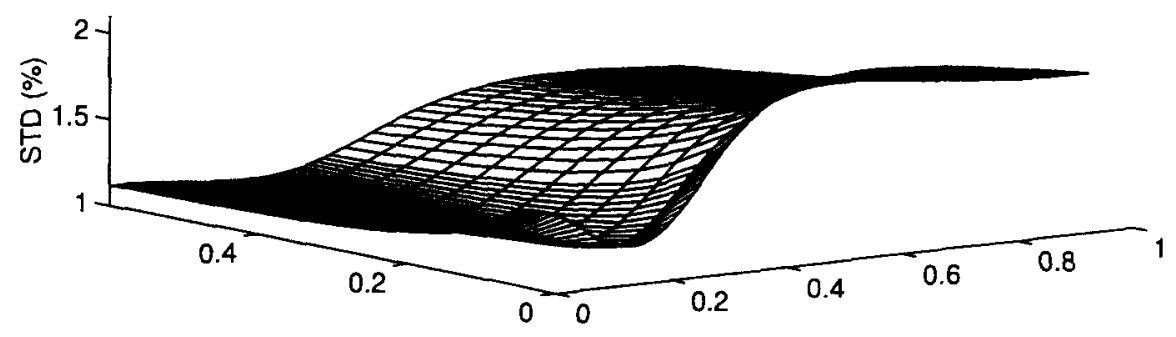

Acceleration of Top Sub Floor

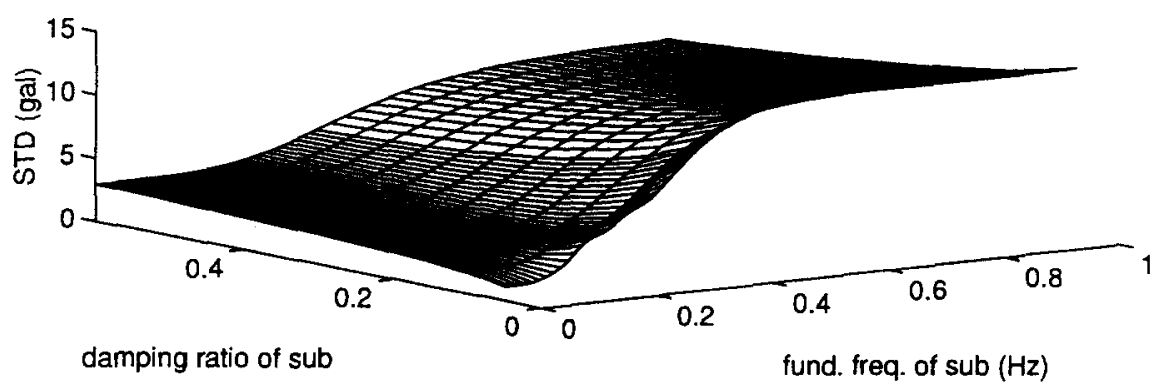

Fig. 10. Stand and deviation of response for mega-sub-controlled building. 

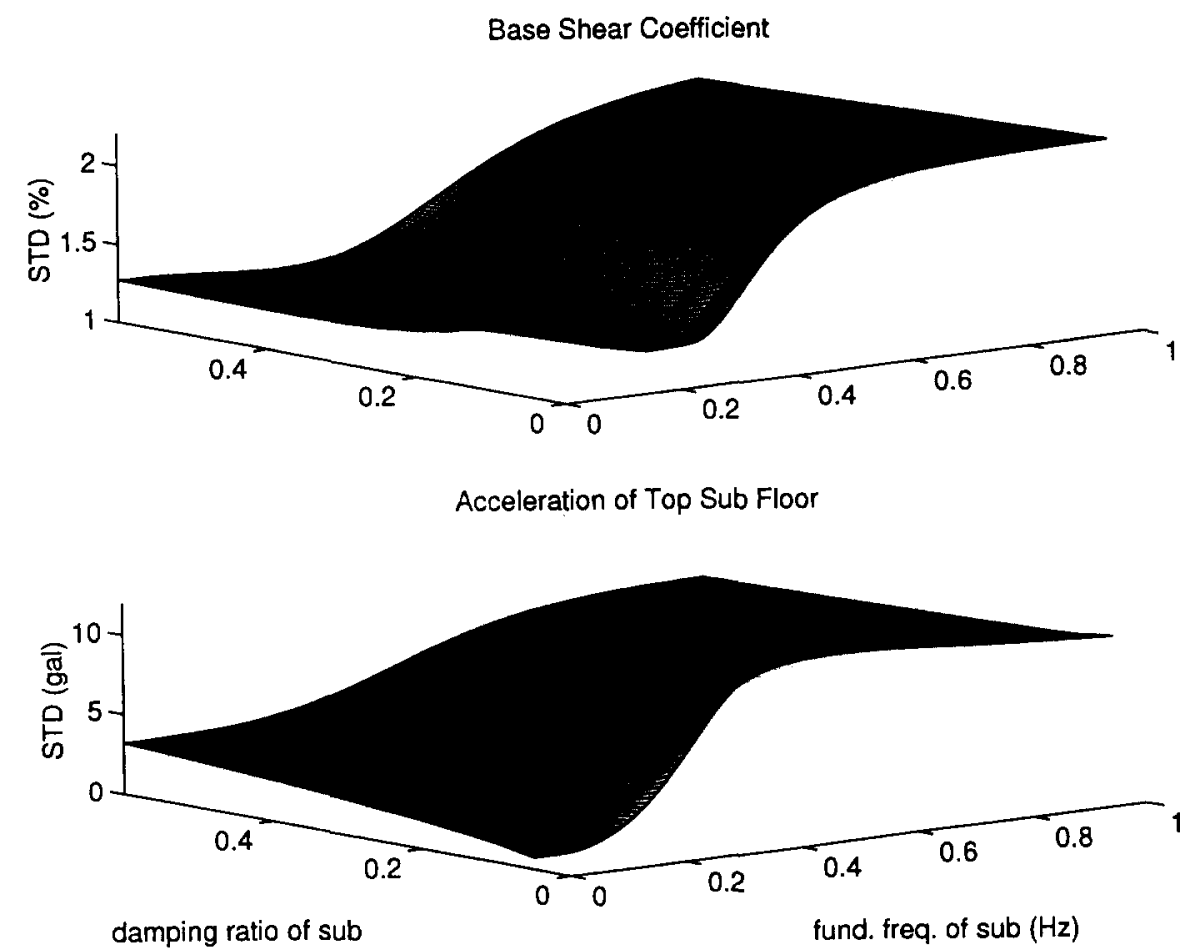

Fig. 11. Standard deviation of response for isolated mega-sub-controlled building.

values resulting in the minimum base shear coefficient of the mega-structure $\left(\omega_{\mathrm{s} 1}=0.2314 \mathrm{~Hz}, h_{\mathrm{s}}=0.3\right.$ and $\omega_{\mathrm{s} 1}=$ $0.2785 \mathrm{~Hz}$ and $h_{\mathrm{s}}=0.15$ ) can lead to a very small acceleration response of the top sub-floor as well. Therefore, those parameter values are selected for this example simulation.

The numerical simulation of the isolated mega-subcontrolled building is conducted to verify the accuracy of the STD of the target responses obtained through the random vibration theory and the validity of the assumptions made during the process. The contribution of the higher order terms in the drag force equation [eqn (13)] have been assumed to be small enough to neglect in order to linearize the drag force equation. This assumption is proved by comparing the response time histories with the effect of higher order terms to those

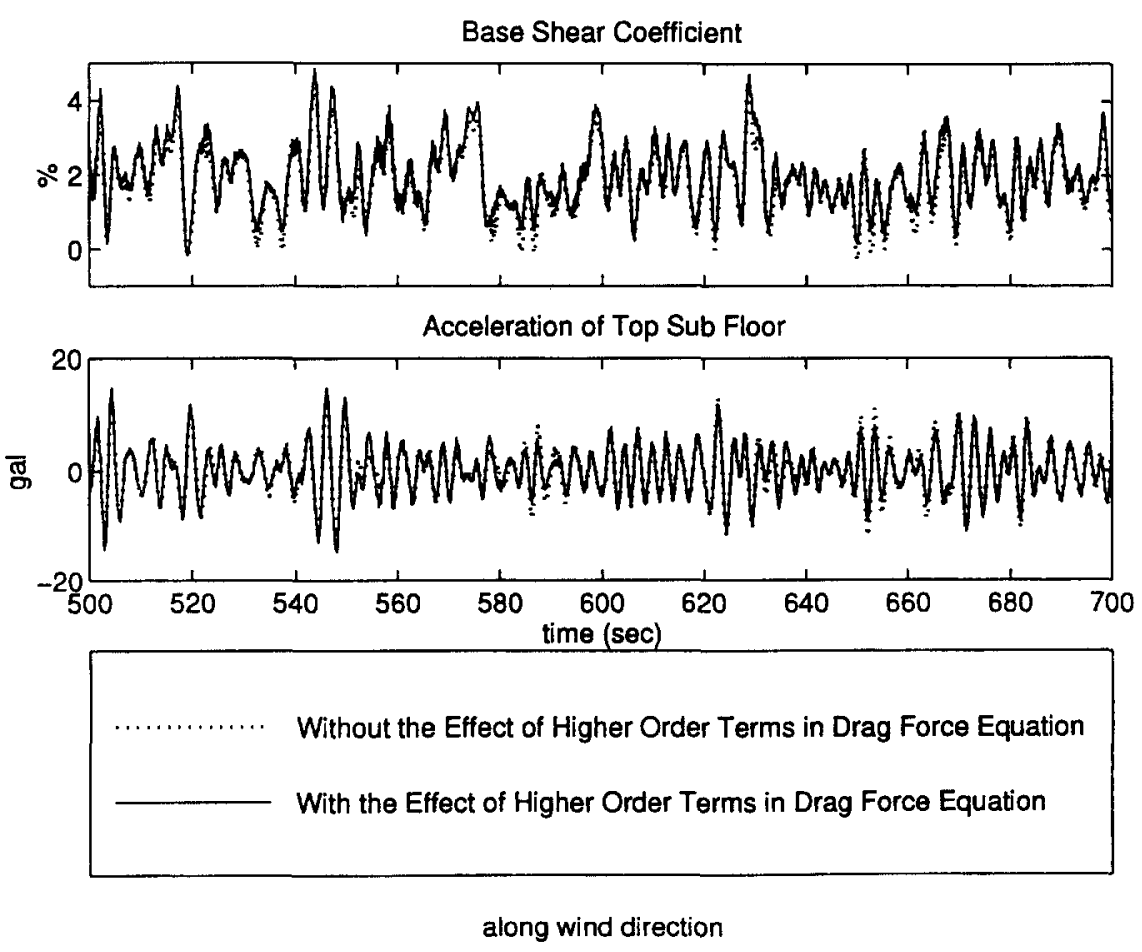

Fig. 12. Time history of building response with and without the effect of higher order terms in drag force equation. 
without as shown in Fig. 12, in which almost no difference is observed. The response time histories of this isolated mega-sub-controlled building are also simulated under the effective drag force. Taking the average of 20 samples, the STD of the displacement response of the bottom mega-floor is $0.3572 \mathrm{~cm}$, and that of the acceleration response of the top sub-floor is $4.0424 \mathrm{gal}\left(1 \mathrm{gal}=1 \mathrm{~cm} \mathrm{~s}^{-2}\right)$. Excellent agreement has been observed between these values and those obtained from the random vibration theory, which are $0.3758 \mathrm{~cm}$
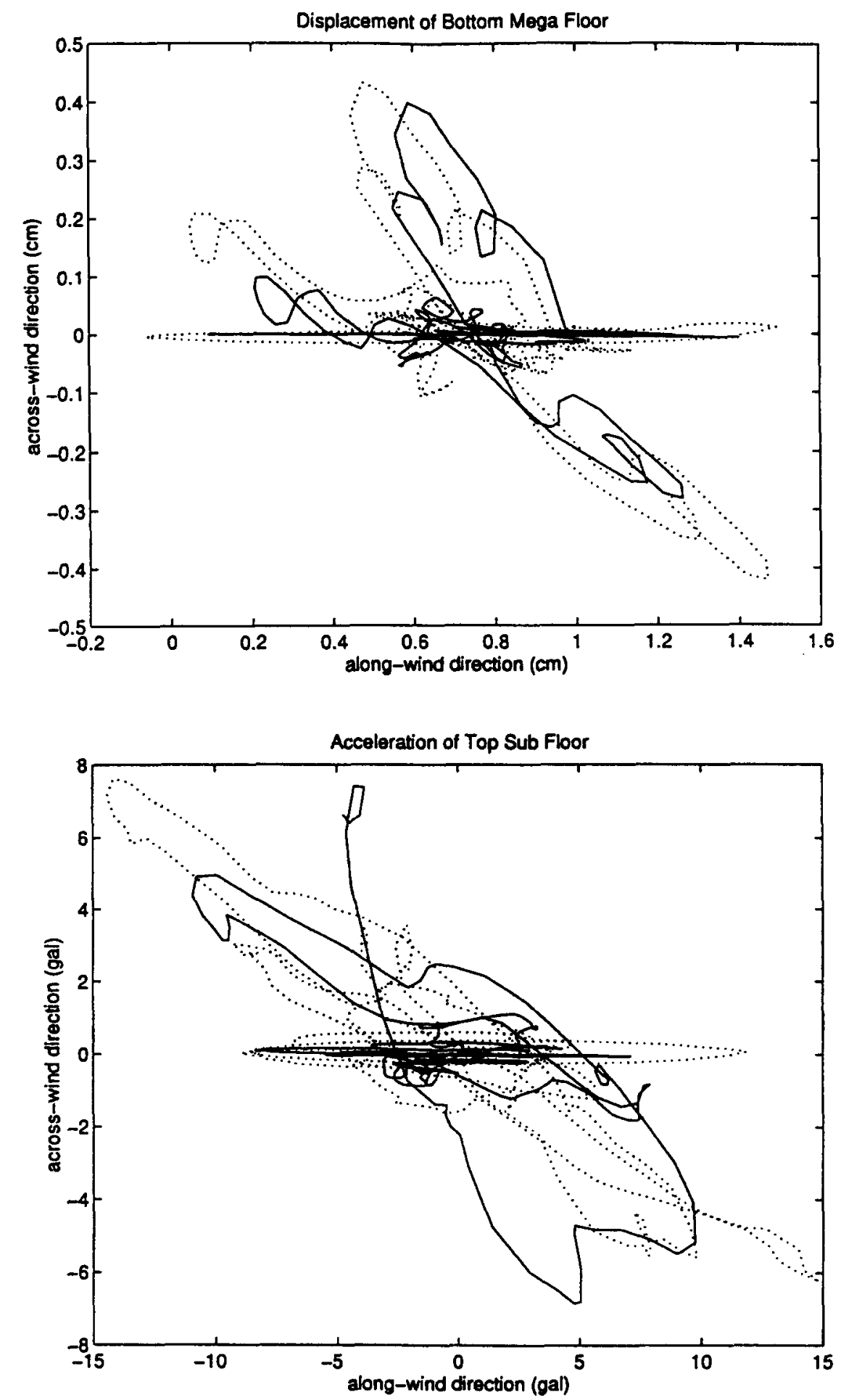

\section{Isolated Mega-Sub Control Mega-Sub Control}

Fig. 13. Response trajectory of mega-sub-controlled and isolated mega-sub-controlled buildings. 
and $4 \cdot 2935$ gal, respectively. Therefore, the accuracy of both random vibration theory in the frequency domain and numerical simulation in the time domain are validated by each other. Using a larger sample size in the numerical simulation, those averages are expected to be even closer to the values obtained from the random vibration theory.

Also of importance is the investigation of the dynamic behaviors of the mega-sub-building with different sub-structure configurations. Figure 13 shows the comparison of the trajectories of the target responses between the mega-sub-controlled and isolated megasub-controlled buildings. Both configurations result in similar trends and peak building responses. The peak values of the inter-story drifts of the mega-structure and acceleration of sub-floors are tabulated in Table 1 for both configurations. Again, comparable effectiveness has been observed in both configurations.

\section{CONCLUSION}

The design procedure of a mega-sub-controlled building under wind excitation is developed with the application of random vibration theory which takes into consideration the dynamic characteristics of both landing and structures in the analysis. Two structural configurations with the mega-sub-control mechanism are suggested: one has sub-structures of shear-building type and the other has the base-isolated sub-structures. Example buildings with these configurations are considered to demonstrate the design procedure, and the optimal values of the dynamic parameters for both configurations are successfully obtained. The comparison between the STD of the target responses obtained from the random vibration theory and numerical simulation shows an excellent agreement. It is concluded that:

(1) the random vibration theory is suitable for design of the mega-sub-controlled buildings under wind excitations;

(2) the mega-sub-control is robust;

(3) similar effectiveness of the mega-sub-control method is demonstrated through the numerical simulation for both sub-structure configurations. The optimal parameter values required for the vibration control purpose are easy to realize through the base isolation of the sub-structures.

\section{ACKNOWLEDGEMENT}

This work was supported by the National Science Foundation under grant no. CMS-9501796 with Dr N. Sabadell as the Program Director. The authors deeply appreciate her encouragement for this work.

\section{REFERENCES}

1. Feng, M. Q. and Mita, A., Vibration of tall buildings using mega-sub configuration. Journal of Engineering Mechanics ASCE, 1995, 121(10).

2. Chai, W. and Feng, M. Q., Vibration control of super tall buildings subjected to wind loads. International Journal of Nonlinear Mechanics (in press).

3. Paz, M., Structural Dynamics, 3rd edn. Van Nostrand-Reinhold, New York, 1991.

4. Simiu, E. and Scanlan, R., Wind Effect on Structure, 2nd edn. Wiley, New York, 1986.

5. Vaicaitis, R., Shinozuka, M. and Takeno, M., Parametric study of wind load on structure. Journal of Structural Division ASCE, 1973, 99(ST3), 453-468.

6. Vaicaitis, R., Shinozuka, M. and Takeno, M., Response analysis of tall buildings to wind loadings. Journal of Structural Division ASCE, 1975, 101(ST3), 585-600.

7. Billah, K., A study of vortex-induced vibration. Ph.D. thesis, Princeton University, 1989.

8. Goswami, I., Scanlan, R. and Jones, N., Vortexinduced vibration of circular cylinders. Part I: Experimental data. Journal of Engineering Mechanics ASCE, 1993, 119(11), 2270-2287.

9. Goswami, I., Scanlan, R. and Jones, N., Vortexinduced vibration of circular cylinders. Part II: New model. Journal of Engineering Mechanics ASCE, 1993, 119(11), 2288-2302.

10. Shinozuka, M. and Deodatis, G., Stochastic process models for earthquake ground motion. Probabilistic Engineering Mechanics, 1988, 3(3), 114-123.

11. Davenport, A. G., The application of statistical concepts to wind loading of structures. Proceedings, Institution of Civil Engineers, 1961, 19, 449-472.

12. Davenport, A. G., The response of slender line-like structures to a gusty wind. Proceedings, Institution of Civil Engineers, 1962, 23, 389-408.

13. Chai, W., Mega-sub control of high-rise buildings. Ph.D. thesis, University of California, Irvine, 1996. 